\title{
Dense ferroelectric-ferroelastic domain structures in rhombohedral PMN-28PT single crystals
}

Cite as: Appl. Phys. Lett. 116, 182901 (2020); doi: 10.1063/5.0008522

Submitted: 23 March 2020 - Accepted: 16 April 2020 .

Published Online: 4 May 2020

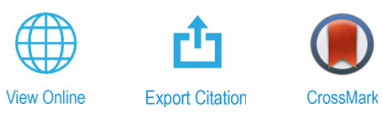

A. D. Ushakov, , a) (D) A. P. Turygin, ${ }^{1}$ (D) A. R. Akhmatkhanov, ${ }^{1}$ (D) D. O. Alikin, ${ }^{1}$ (D) Q. Hu, ${ }^{2}$ (D) X. Liu, ${ }^{2}$ (D) Y. Zhao, Z. Xu, ${ }^{2}$ X. Wei, ${ }^{2}$ (D) and V. Ya Shur ${ }^{1, b)}$ (iD

\begin{abstract}
AFFILIATIONS
${ }^{7}$ School of Natural Sciences and Mathematics, Ural Federal University, 620000 Ekaterinburg, Russia

${ }^{2}$ Electronic Materials Research Laboratory, Key Laboratory of the Ministry of Education and International Center

for Dielectric Research, Xi'an Jiaotong University, 710049 Xi'an, China
\end{abstract}

a) Author to whom correspondence should be addressed: bddah@ya.ru

${ }^{b)}$ Electronic mail: vladimir.shur@urfu.ru

\begin{abstract}
We have studied a dense domain structure (DDS) appearing during polarization reversal in rhombohedral (111)-cut lead magnesium niobate-lead titanate single crystals. The complicated shape of the switching current was explained by means of non-uniform motion of the boundary between the DDS and $\boldsymbol{c}$-domains using comparative analysis of the optical current and kinetic map. 2D-vector piezoresponse force microscopy revealed that DDS represented needle-like $\boldsymbol{a}$-domains inside the $\boldsymbol{a}$-domains with different vertical orientations of spontaneous polarization.
\end{abstract}

Published under license by AIP Publishing. https://doi.org/10.1063/5.0008522

Interest in a relaxor-based ferroelectric-ferroelastic lead magnesium niobate-lead titanate (PMN-PT) solid solution is growing due to its low value of dielectric losses and one of the highest piezoelectric performances. ${ }^{1-4}$ Recently, second harmonic generation measurements of crystal powder of tetragonal PMN-PT have revealed its promising nonlinear-optical properties. ${ }^{5}$ Since it is impossible to realize the phase matching conditions in PMN-PT, the creation of a precise periodical domain structure for the realization of the quasi-phase matching effect is necessary for effective second harmonic generation. ${ }^{6}$ A deeper understanding of the physical fundamentals of domain structure evolution and polarization reversal process in PMN-PT is thereby crucial to create the domain patterns with a controllable geometry. The increase in the domain wall concentration by creation of the dense domain structures (DDSs) represents domain wall engineering, which is used, e.g., for improving the PMN-PT piezoelectric performance. ${ }^{8}$ Thus, the investigation of DDS formation is important. The statistical analysis of the elementary domain structure evolution events obtained by optical imaging and switching current recording over the whole length scale was applied in barium titanate $\mathrm{BaTiO}_{3}$ crystals. ${ }^{10}$ The simultaneous recording of the instantaneous domain images and current (Barkhausen pulses) was successfully applied by Miller ${ }^{11}$ and later was used for the investigation of various ferroelectric materials. ${ }^{12-15}$
Earlier, we studied the domain structure evolution in rhombohedral [111]-oriented PMN-PT single crystals during polarization reversal. ${ }^{16}$ We used the direct optical observation of the domain structure during polarization reversal and proposed the following domain structure evolution stages: (1) nucleation and growth of $\boldsymbol{a}$-domains and domains with charged domain walls at the intersection of two $\boldsymbol{a}$-domains $(2 \boldsymbol{a}$-CDW) representing the creation of the so-called DDS and (2) consequent growth of $\boldsymbol{c}$-domains. It is worth noting that the term "dense" does not mean "extremely dense" but is introduced in order to separate and mark the domain structure types. The decrease in the optical contrast and intensity as a result of DDS formation was observed. However, the geometry of DDS remained unknown. In this work, we analyze the formation and geometry of DDS by complementary in situ optical imaging of domain structure evolution, switching current measurements, and vector piezoresponse force microscopy (PFM).

PMN-PT single crystals were grown by the modified Bridgman technique in Xi'an Jiaotong University. ${ }^{18}$ The studied samples belonged to a rhombohedral symmetry at room temperature and represented 0.5 -mm-thick plates of $6 \times 4 \mathrm{~mm}^{2}$ size oriented by means of rotating orientation $\mathrm{x}$-ray diffraction ${ }^{19}$ and cut perpendicular to the [111] direction with the side surfaces parallel to $(11 \overline{2})$ and $(1 \overline{1} 0)$ 
TABLE I. The notations for the orientations of the spontaneous polarization vector for the rhombohedral cell in the [111]-cut samples.

\begin{tabular}{lccc}
\hline \hline $\begin{array}{l}\text { Spontaneous } \\
\text { polarization vector }\end{array}$ & Notation & $\begin{array}{c}\text { Spontaneous } \\
\text { polarization vector }\end{array}$ & Notation \\
\hline$[111]$ & $c \uparrow$ & {$[\overline{1} \overline{1} 1]$} & $\mathrm{a}_{1} \downarrow$ \\
{$[11 \overline{1}]$} & $\mathrm{a}_{1} \uparrow$ & {$[1 \overline{1} \overline{1}]$} & $\mathrm{a}_{2} \downarrow$ \\
{$[\overline{1} 11]$} & $\mathrm{a}_{2} \uparrow$ & {$[\overline{1} 1 \overline{1}]$} & $\mathrm{a}_{3} \downarrow$ \\
{$[1 \overline{1} 1]$} & $\mathrm{a}_{3} \uparrow$ & {$[\overline{111}]$} & $\boldsymbol{c} \downarrow$ \\
\hline \hline
\end{tabular}

planes. The mechanochemical polishing was realized using a silica compound SF1 suspension by a PM5 polishing machine (Logitech Ltd., Glasgow, UK). The as-grown sample was annealed at $750^{\circ} \mathrm{C}$ for $10 \mathrm{~h}$ to release the stresses produced by crystal growth and mechanical treatment. ${ }^{20}$ The crystal composition was confirmed by dielectric measurements using the dependence of temperature of maximum permittivity $T_{m}$ on the composition of $(1-x) \mathrm{PMN}-x \mathrm{PT}$ according to the following formula (see the supplementary material for dielectric measurements) $)^{21,22}$

$$
x=\frac{T_{m}+12^{\circ} \mathrm{C}}{527^{\circ} \mathrm{C}} .
$$

The visualization of domain kinetics during the polarization reversal was carried out using an experimental setup based on a polarizing optical microscope LMA10 (CarlZeiss AG, Oberkochen, Germany). The domain kinetics images were recorded synchronously with the switching current data. A high-speed CMOS camera Mini UX100 (Photron Ltd., Tokyo, Japan) was used to record a video of the domain kinetics with the frame rate up to $1000 \mathrm{~Hz}$ and $1280 \times 1024$ pixels resolution corresponding to the $2 \mu \mathrm{m}$ spatial resolution. The voltage pulses were generated by a multifunctional data acquisition board PCI-6251 (National Instruments Corp., Austin, USA) and amplified using a high-voltage amplifier TREK 677B (Trek Inc., Lockport, USA). The op-amp-based feedback ammeter allowed gathering the switching current. A saturated aqueous solution of lithium chloride was used as the liquid electrode. The top and bottom surfaces of the crystal were uniformly covered by the electrolyte. The size of the switching area was restricted at the top surface by a hole in a Petri dish. The polarization reversal was carried out at room temperature. The details of the experimental setup can be found in Refs. 16 and 23-25 and in the supplementary material. The optical image processing was performed using Fiji ${ }^{26}$ distribution of ImageJ. ${ }^{2}$

The unipolar trapezoidal electric field pulse was used for the polarization reversal. ${ }^{16}$ The pulse amplitude was $275 \mathrm{~V} / \mathrm{mm}$, the pulse duration was $1.5 \mathrm{~s}$, and the rise and fall time was $100 \mathrm{~ms}$. The sample was pre-poled before polarization reversal experiments by the application of a trapezoidal field pulse with an amplitude of $300 \mathrm{~V} / \mathrm{mm}$ and a duration of $3 \mathrm{~s}$. This procedure allows obtaining the domain structure with the preferred orientation of $\boldsymbol{c}$-domains (here, $\boldsymbol{c} \uparrow$-domains) and small amount of $\boldsymbol{a} \uparrow$-domains.

The $R 3 m$ rhombohedral-symmetry ferroelectric single crystal possesses eight possible directions of spontaneous polarization. ${ }^{28}$ The notations for the orientation of the spontaneous polarization vector used in this paper are summarized in Table I.
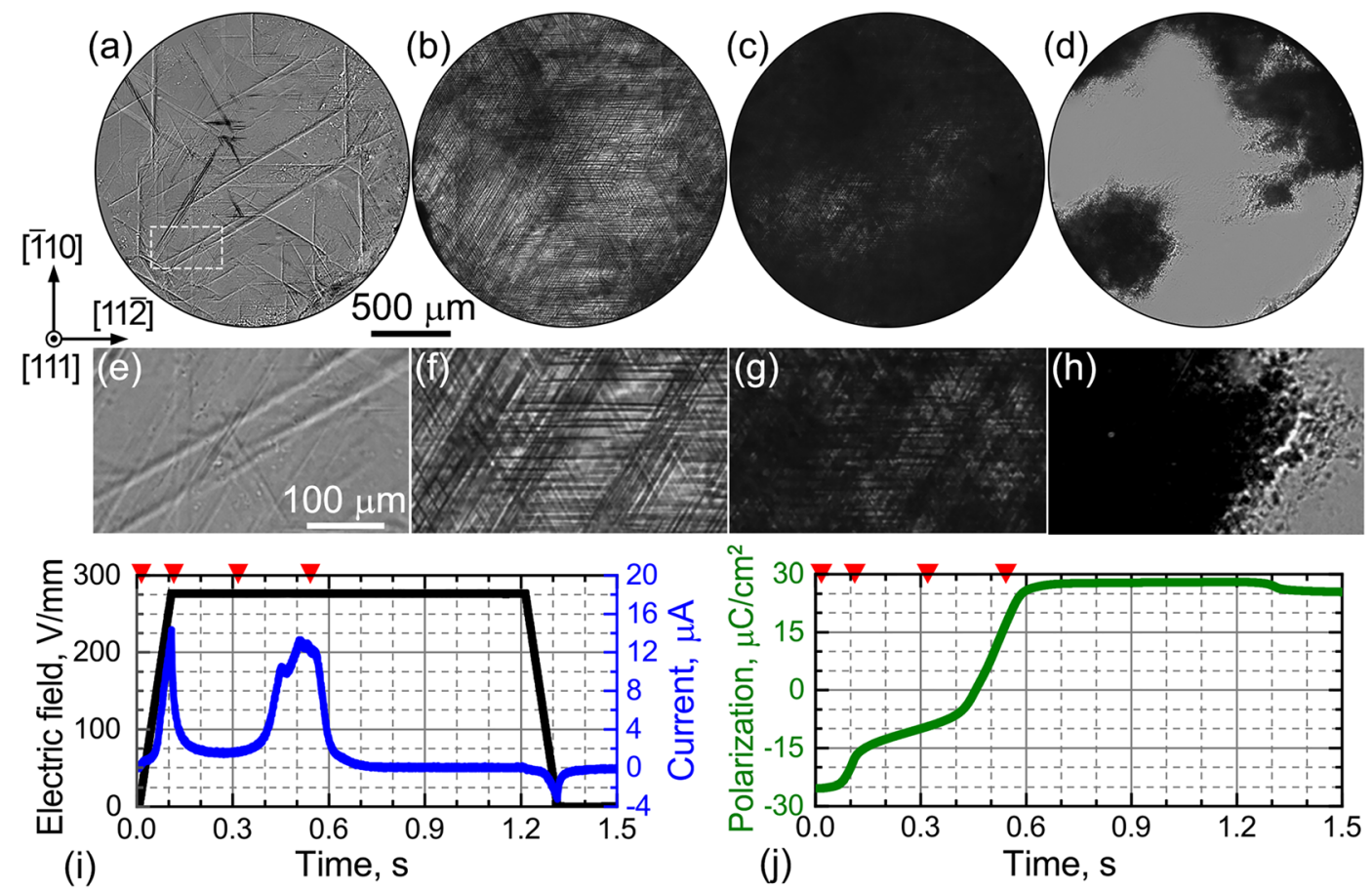

FIG. 1. (a)-(d) Optical images of the domain patterns recorded at (a) 0.003 , (b) 0.108 , (c) 0.318 , and (d) $0.545 \mathrm{~s}$. (e)-(h) Zoomed optical images of the region marked with the dotted frame in (a). The red cursors in (i) and (j) point the moment of time, when the images (a)-(d) were obtained. Time dependences of the (i) electric field and switching current, and (j) polarization obtained during polarization reversal. Multimedia view: https://doi.org/10.1063/5.0008522.1 
The domain structure at the surface appeared after polarization reversal was visualized using a scanning probe microscope Asylum MFP-3D (Oxford Instruments, Abingdon, UK). The proprietary technique Dual AC Resonance Tracking (DART) for PFM traced the frequency of the first flexural contact resonance. ${ }^{29}$ Commercial HA_NC tips with $\mathrm{W}_{2} \mathrm{C}$ coating (ScanSens, Bremen, Germany) possessing a nominal curvature radius of $35 \mathrm{~nm}$, a $235 \mathrm{kHz}$ frequency of the free cantilever resonance, and a spring constant of $12 \mathrm{~N} / \mathrm{m}$ were used. A $750 \mathrm{mV}$ AC voltage was applied during PFM measurements.

The typical optical images of the dense domain structure (DDS) evolution obtained at the stage of nucleation, growth, and intersection of $\boldsymbol{a} \uparrow$-domains ${ }^{16}$ during polarization reversal are shown in [Figs. 1(a)-1(d), Multimedia view]. The enlarged images of the region marked in Fig. 1(a) are shown in Figs. 1(e)-1(h). The corresponding field pulse and time dependences of the switching current and polarization are shown in Figs. 1(i) and 1(j). It was proposed that the intersection of $\boldsymbol{a} \uparrow$-domains led to the formation of the DDS consisting of needle-like domains with charged walls $(2 a$-CDW $) .{ }^{16}$ During the rise of the field, the nucleation and elongation of the DDS [Fig. 1(g) and 1(h)] impeded an optical observation of the forming domain pattern due to the light dissipation. The low intensity of the DDS is explained by using the optical objective with a low numerical aperture $(\mathrm{NA}=0.1)$. The high-NA objective [Figs. S2(a) and S2(b)] or the back focal plane mode [Fig. S2(c)] allows revealing the characteristic orientations of domains within DDS along the $[11 \overline{2}],[1 \overline{21}]$, and $[\overline{2} 11]$ crystallographic directions (see the supplementary material). The switching current during this stage has a single sharp peak with the maximum coinciding to the field rate change $(0.1 \mathrm{~s})$. High current values were attributed to the rapid growth of $\boldsymbol{a}$-domains and DDS under the increasing external field. It should be noted that the current had a non-zero value in the time range from 0.20 to $0.40 \mathrm{~s}$, but the optical changes could not be observed due to the lack of the optical contrast in the DDS area.

The second stage of the domain structure evolution corresponds to the switching from the DDS into the $\boldsymbol{c} \downarrow$-domain state. The corresponding switching current (from 0.40 to $0.64 \mathrm{~s}$ ) has a complicated shape [Fig. 1(i)]. The main part of the polarization change (about $60 \%)$ was switched at this stage [Fig. 1(j)]. To address the origin of such switching current behavior, we extracted the optical current ${ }^{30}$ [Fig. 2(a)]. The set of instantaneous domain images was processed and analyzed. The optical current was calculated as the difference in the areas occupied by $\boldsymbol{c} \downarrow$-domains in the consequent images

$$
j_{\text {opt. } n}=\frac{A_{n}-A_{n-1}}{\Delta t_{n}},
$$

where $A_{n}$ is the area of $c \downarrow$-domains in the image $n$ and $\Delta t_{n}$ is the fixed time interval between the consequent images.

It is seen that the optical and switching currents are highly correlated [Fig. 2(a)]. The discrepancy of the currents at the beginning of the stage (time interval $0.40-0.45 \mathrm{~s}$ ) can be related to the inability of detecting changes inside the DDS.

The kinetic map ${ }^{31}$ [Fig. 2(b)] representing a set of the consequent positions of the boundary between the DDS and $\boldsymbol{c} \downarrow$-domain area was constructed. The corresponding temporal-color code is presented in Fig. 2(a). The comparison of the optical current and kinetic map allowed finding the relation between the individual

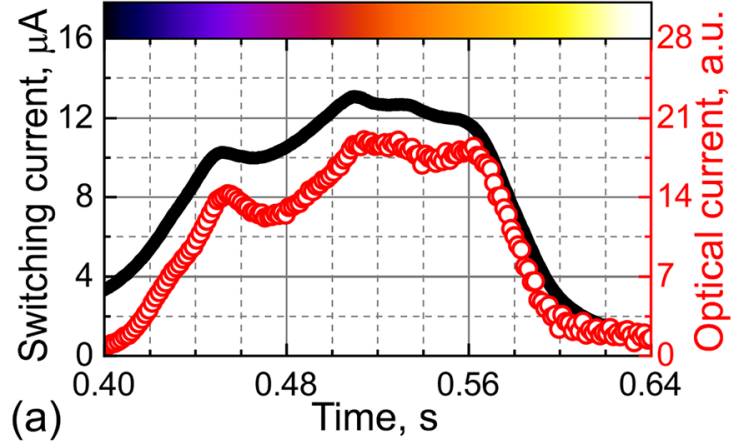

(b)

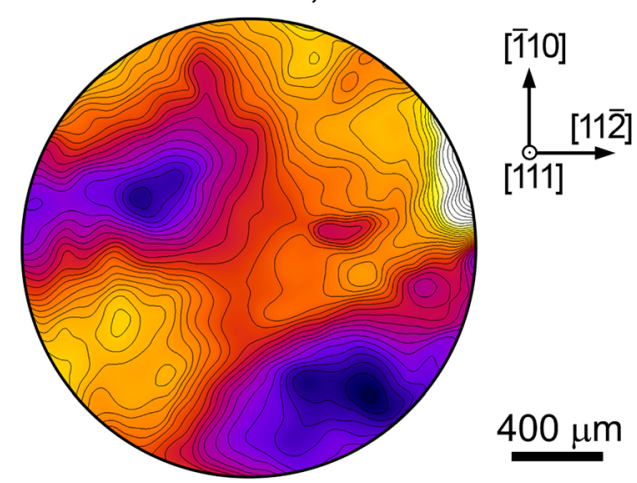

FIG. 2. (a) Comparison of the switching (black line) and optical (red circles) currents and (b) kinetic map within the time range from 0.40 to $0.64 \mathrm{~s}$ of polarization reversal. The kinetic map is the visual interpretation of motion of the boundary between DDS and $\mathrm{c} \downarrow$-domains. The amount of boundaries was decreased for better visual reception. Color corresponds to the timescale in (a).

switching events and the switching current features. It was found that the switching current peaks corresponded to the increase in the boundary motion velocity after merging of the isolated switched areas (see the supplementary material). ${ }^{24,31}$

In order to study the DDS, we implemented vector-PFM measurements [Figs. 3(a)-3(d)]. The combination of the vertical and lateral signals can be used for the reconstruction of the domain structure and polarization orientation in the samples with the known orientation of polar axes. ${ }^{17,32}$ The limited number of possible polarization directions in the studied crystal and scanning along the $[11 \overline{2}]$ direction allowed us to recover the direction of polarization in different domains. The recorded vertical piezoresponse was related solely to out-of-plane polarization, while the in-plane component, the so-called "buckling," 33 was found to be negligible (see the supplementary material). The optical image of the area inspected by PFM is shown in Fig. 3(e). The analysis of both vertical and lateral amplitudes of the piezoresponse signal [Figs. 3(a) and 3(c)] allowed distinguishing $\boldsymbol{c}$ and $\boldsymbol{a}$-domains. The c-domains had a maximal response in the vertical signal, while $\boldsymbol{a}$-domains-in the lateral one due to the different amplitudes of the polarization vector component in the (111) plane. The phase difference of the vertical signal provided the ability to differentiate the orientation of the vertical component ( $\uparrow$ or $\downarrow$ ) of spontaneous polarization. The types of $\boldsymbol{a}$-domains were separated by the difference of the lateral phase signal. The resulted scheme based on the combination of all obtained PFM signals [Fig. 3(f)] contains eight different 

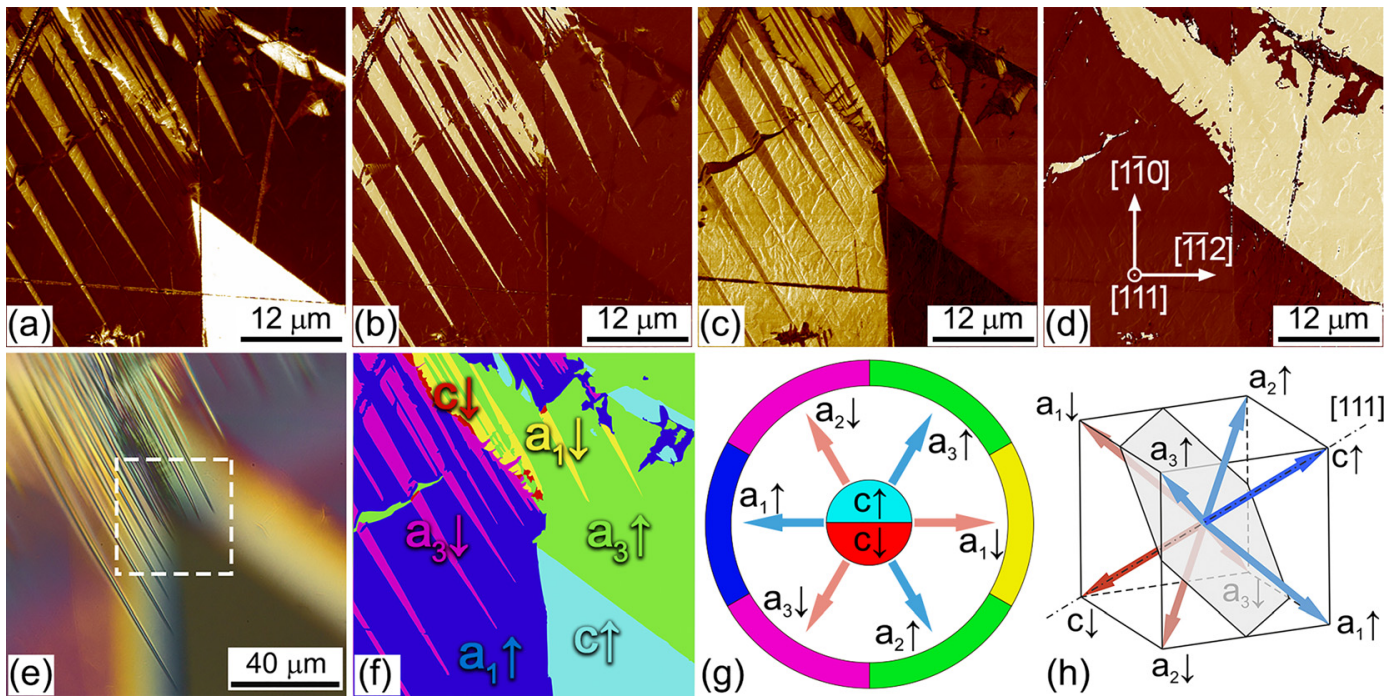

FIG. 3. Intersection of two a-domains studied by PFM. (a) and (b) Vertical and (c) and (d) lateral, (a) and (c) amplitude and (b) and (d) phase PFM signals obtained from the sample region marked in (e) polarized-light optical microscopy image. (f) Schematic image of the domain structure from (a)-(d) with the polarization orientation coded by ( $g$ ) color wheel. The scanning direction is along [112]. See the 3D scheme of the investigated area in the supplementary material.

domain types coded with a color wheel [Fig. 3(g)]. These measurements revealed that the DDS consisted of needle-like $\boldsymbol{a} \downarrow$-domains inside the $\boldsymbol{a} \uparrow$-domains (or vice versa, depending on the initial polarization orientation). In such a configuration of the domain structure, domain walls between $\boldsymbol{a} \downarrow$-domains and $\boldsymbol{a} \uparrow$-domains were vertical: (1) 0$),(0 \overline{1} 1)$, and $(10 \overline{1})$. The $3 \mathrm{D}$ scheme of the investigated domain structure can be seen in the supplementary material. The micrometersized $\boldsymbol{c} \downarrow$-domains at the intersection area between $\boldsymbol{a} \downarrow$-domains are expected to be the nuclei for the consequent switching from DDS to the $\boldsymbol{c} \downarrow$-domain state.

In summary, we studied in detail the DDS in a (111)-cut rhombohedral PMN-PT crystal appearing during polarization reversal. The comparative analysis of the optical current and kinetic map allowed attributing the complex switching current shape to increase in the boundary motion velocity after merging of the isolated switched areas. 2D-vector piezoresponse force microscopy revealed that this structure type represented the needle-like $\boldsymbol{a}$ domains inside the $\boldsymbol{a}$-domains of different vertical orientations of spontaneous polarization.

See the supplementary material for the details of the sample characterization, experimental setup, optical current, and kinetic map estimation, and PFM measurements of the DDS.

The equipment of the Ural Center for Shared Use "Modern Nanotechnology" UrFU was used. This work has been supported in part by the Ministry of Science and Higher Education of the Russian Federation under Project No. 3.9534.2017/8.9, by the Government of the Russian Federation (Act 211, Agreement No. 02.180 A03.21.0006) and by RFBR (Grant No. 17-52-80116BRICS_a). This work was supported by the NSFC projects (Grant No. 51761145024), Shaan Xi province Project (Nos. 2017 ktpt-21 and 2018TD-024), and the 111 Project under Grant No. B14040.
The data that support the findings of this study are available within the article and its supplementary material.

\section{REFERENCES}

${ }^{1}$ S. E. Park and T. R. Shrout, J. Appl. Phys. 82, 1804 (1997).

${ }^{2}$ R. Zhang, B. Jiang, and W. Cao, J. Appl. Phys. 90, 3471 (2001).

${ }^{3}$ F. Li, M. J. Cabral, B. Xu, Z. Cheng, E. C. Dickey, J. M. Lebeau, J. Wang, J. Luo, S. Taylor, W. Hackenberger, L. Bellaiche, Z. Xu, L. Chen, T. R. Shrout, and S. Zhang, Science 364, 264 (2019).

${ }^{4}$ S. Zhang and F. Li, J. Appl. Phys. 111, 031301 (2012).

${ }^{5}$ Y. Zhao, X. Liu, B. Li, Q. Hu, Y. Zhuang, X. Fu, P. Luan, W. Zhao, Y. Liu, Z. Li, G. Zhang, V. Ya. Shur, Z. Xu, and X. Wei, Ferroelectrics 542, 112 (2019).

${ }^{6}$ V. Ya. Shur, E. L. Rumyantsev, E. V. Nikolaeva, E. I. Shishkin, R. G. Batchko, M. M. Fejer, and R. L. Byer, Ferroelectrics 257, 191 (2001).

7S. Wada, S. E. Park, L. E. Cross, and T. R. Shrout, Ferroelectrics 221, 147 (1999).

${ }^{8}$ Z. Zhang, J. Xu, L. Yang, S. Liu, J. Xiao, R. Zhu, X. Li, X. Wang, and H. Luo, J. Appl. Phys. 125, 034104 (2019).

${ }^{9}$ X. Ding, Z. Zhao, T. Lookman, A. Saxena, and E. K. H. Salje, Adv. Mater. 24, 5385 (2012).

${ }^{10}$ B. Casals, G. F. Nataf, D. Pesquera, and E. K. H. Salje, APL Mater. 8, 011105 (2020).

${ }^{11}$ R. C. Miller, Phys. Rev. 111, 736 (1958).

${ }^{12}$ V. Ya. Shur, E. V. Nikolaeva, E. L. Rumyantsev, E. I. Shishkin, A. L. Subbotin, and V. L. Kozhevnikov, Ferroelectrics 222, 323 (1999).

${ }^{13}$ V. Ya. Shur, E. L. Rumyantsev, D. V. Pelegov, V. L. Kozhevnikov, E. V. Nikolaeva, E. I. Shishkin, A. P. Chernykh, and R. K. Ivanov, Ferroelectrics 267, 347 (2002).

${ }^{14}$ D. C. Lupascu, V. Ya. Shur, and A. G. Shur, Appl. Phys. Lett. 80, 2359 (2002).

${ }^{15}$ I. S. Baturin, M. V. Konev, A. R. Akhmatkhanov, A. I. Lobov, and V. Ya. Shur, Ferroelectrics 374, 136 (2008).

${ }^{16}$ A. D. Ushakov, A. A. Esin, A. R. Akhmatkhanov, Q. Hu, X. Liu, Y. Zhao, A. A. Andreev, X. Wei, and V. Ya. Shur, Appl. Phys. Lett. 115, 102903 (2019).

${ }^{17}$ S. V. Kalinin, B. J. Rodriguez, S. Jesse, J. Shin, A. P. Baddorf, P. Gupta, H. Jain, D. B. Williams, and A. Gruverman, Microsc. Microanal. 12, 206 (2006).

${ }^{18}$ H. Luo, G. Xu, H. Xu, P. Wang, and Z. Yin, Jpn. J. Appl, Phys., Part 1 39, 5581 (2000).

${ }^{19}$ F. Li, L. Jin, Z. Xu, and Z. Guo, Rev. Sci. Instrum. 80, 085106 (2009). 
${ }^{20}$ Y. Zhao, S. Wang, X. Fu, Y. Zhuang, R. Yang, Z. Yang, Z. Li, Z. Xu, and X. Wei, J. Appl. Phys. 123, 084104 (2018).

${ }^{21} \mathrm{~F}$. Li, S. Zhang, Z. Xu, X. Wei, J. Luo, and T. R. Shrout, J. Appl. Phys. 108, 034106 (2010).

${ }^{22}$ M. Davis, "Phase Transitions, Anisotropy and Domain Engineering: The Piezoelectric Properties of Relaxor-Ferroelectric Single Crystals," Ph.D. thesis (Ecole Polytechnique Federale de Lausanne, 2006).

${ }^{23}$ A. A. Esin, A. R. Akhmatkhanov, and V. Ya. Shur, Appl. Phys. Lett. 114, 092901 (2019).

${ }^{24}$ V. Ya. Shur, A. A. Esin, M. A. Alam, and A. R. Akhmatkhanov, Appl. Phys. Lett. 111, 152907 (2017).

${ }^{25}$ A. D. Ushakov, A. A. Esin, A. R. Akhmatkhanov, Q. Hu, X. Liu, Y. Zhao, X. Wei, and V. Ya. Shur, Appl. Phys. Lett. 113, 112902 (2018).

${ }^{26}$ J. Schindelin, I. Arganda-Carreras, E. Frise, V. Kaynig, M. Longair, T. Pietzsch, S. Preibisch, C. Rueden, S. Saalfeld, B. Schmid, J. Y. Tinevez, D. J. White, V.
Hartenstein, K. Eliceiri, P. Tomancak, and A. Cardona, Nat. Methods 9, 676 (2012).

${ }^{27}$ C. T. Rueden, J. Schindelin, M. C. Hiner, B. E. DeZonia, A. E. Walter, E. T. Arena, and K. W. Eliceiri, BMC Bioinf. 18, 529 (2017).

${ }^{28}$ I. S. Zheludev, Solid State Phys. 26, 429 (1971).

${ }^{29}$ B. J. Rodriguez, C. Callahan, S. V. Kalinin, and R. Proksch, Nanotechnology 18, 475504 (2007).

${ }^{30}$ V. Ya. Shur, E. V. Nikolaeva, E. I. Shishkin, V. L. Kozhevnikov, A. P. Chernykh, K. Terabe, and K. Kitamura, Appl. Phys. Lett. 79, 3146 (2001).

${ }^{31}$ A. A. Esin, A. R. Akhmatkhanov, and V. Ya. Shur, Appl. Phys. Lett. 114, 192902 (2019).

${ }^{32}$ L. M. Eng, H.-J. Güntherodt, G. A. Schneider, U. Köpke, and J. Muñoz Saldaña, Appl. Phys. Lett. 74, 233 (1999).

${ }^{33}$ E. Soergel, J. Phys. D. Appl. Phys. 44, 464003 (2011). 